\title{
División e historia del currículo
}

\section{Division and history of the curriculum}

\section{Divisão e história do currículo}

Gladys J. Negrete-Chauca ${ }^{\text {I }}$

gladysnegretech@hotmail.com

Eleana I. Rugel-Jiménez II

eleanarugeljimenez@hotmail.com

Alfonsina B. Hernández-Valencia III

alfonsina_27_hernandez@hotmail.com

Viviana F. Pinos-Medrano IV

viviana150987@hotmail.com

Recibido: 10 de enero de 2019 *Aceptado: 08 de febrero de $2019 *$ Publicado: 05 de abril de 2019

I. Profesora de Segunda Enseñanza Especialización Lengua Inglesa y Lingüística; Licenciada en Ciencias de la Educación mención Informática Educativa; Universidad de Guayaquil; Guayaquil, Ecuador.

II. Licenciada en Ciencias de la Educación Especialización Fisico-Matematica; Profesora de Segunda Enseñanza en la Especialización de Físico Matemáticas; Universidad de Guayaquil; Guayaquil, Ecuador.

III. Licenciada en Ciencias de la Educación Mención Literatura y Español; Profesor de Segunda Enseñanza Especialización: Literatura y Castellano; Universidad de Guayaquil; Guayaquil, Ecuador.

IV. Magister en Diseño Curricular; Diploma Superior en Diseño Curricular por Competencias; Licenciada en Turismo y Hotelería; Universidad de Guayaquil; Guayaquil, Ecuador. 


\title{
Resumen
}

El presente artículo traza miramientos o consideraciones generales sobre la jerarquía del currículo en los procesos y técnicas educativas sobre varias concepciones que existen en la conceptualización del currículo y las hipótesis curriculares que fundamentan los diseños y perfeccionamiento curricular y los desafíos para educar a niños, jóvenes y adultos con capacidades, aptitudes competencias e idoneidades, responsables en el adelanto de las sociedades en las que se examine, investigue y se vivencie los derechos humanos para todos y todas.

Palabras claves: Pedagogía; Logros; Eficiencia.

\begin{abstract}
The present article traces considerations or general considerations on the hierarchy of the curriculum in the educational processes and techniques on various conceptions that exist in the conceptualization of the curriculum and the curricular hypotheses that underlie curriculum designs and refinements and the challenges to educate children, young people and adults with abilities, aptitudes, competencies and skills, responsible for the advancement of societies in which human rights are examined, investigated and lived for all.
\end{abstract}

Key words: Pedagogy; Achievements; Efficiency.

\section{Resumo}

O presente artigo traça considerações ou considerações gerais sobre a hierarquia do currículo nos processos e técnicas educacionais sobre as várias concepções que existem na conceituação do currículo e as hipóteses curriculares que fundamentam os projetos e os refinamentos curriculares e os desafios para educar as crianças, os jovens e adultos com habilidades, aptidões, competências e habilidades, responsáveis pelo avanço das sociedades em que os direitos humanos foram examinados, pesquisados e vividos para todos.

Palavras chave: Pedagogia Conquistas Eficiência.

\footnotetext{
417 Vol. 5, núm. 2, abril 2019, pp. 416-440 Gladys J. Negrete-Chauca; Eleana I. Rugel-Jiménez; Alfonsina B. Hernández-Valencia; Viviana F. Pinos-Medrano
} 


\section{Introducción.}

Son todas las experiencias de aprendizaje planeadas y dirigidas por la escuela para alcanzar sus metas educacionales. Ralph Tyler (1949)

Entonces se puede decir que el currículo contiene una serie de objetivos, metas y contenidos empíricos y científicos, organizados de tal manera que lleguen a ser modelos de enseñanza y de aprendizaje, para niños, jóvenes y adultos. Y a la vez contiene patrones de evaluación periódica de sus contenidos, para poder medir el nivel de conocimientos que los estudiantes logren alcanzar en un determinado tiempo de estudio.

El currículo ayuda tanto a docentes como a los estudiantes a desarrollar ciertas habilidades y destrezas, además es un programa global, completo y flexible porque es un conjunto de contenidos y conceptos que se encuentran interrelacionados y estructurados de una forma organizada de valores, normas, elementos culturales, costumbres, creencias con el único fin de producir aprendizajes significativos en todas las personas, para que éstas cambien su manera de pensar y logren desarrollar el pensamiento crítico y creativo.

\section{División del currículo}

El currículo en general se divide en tres grupos:

1- Currículo Vitae.

2- Currículo Educativo.

3- Currículo oculto.

\section{Currículo vitae}

El currículo Vitae fue creado por Leonardo da Vinci en 1482, a pesar de haberse creado muchos siglos atrás, hasta los años de 1940 el currículo no era más que una mera formalidad.

El currículo Vitae a partir de la década de 1950 comenzó a ser entregado en los diferentes sitios que las personas pretendía encontrar trabajo, en este documento se escribían algunos datos

\footnotetext{
418 Vol. 5, núm. 2, abril 2019, pp. 416-440 Gladys J. Negrete-Chauca; Eleana I. Rugel-Jiménez; Alfonsina B. Hernández-Valencia; Viviana F. Pinos-Medrano
} 
importantes de la persona que necesitaba ingresar a trabajar en alguna fábrica o una institución pública.

El origen y progreso del currículo vitae como se menciona al inicio del escrito, aunque en aquel tiempo no era usual ni existía la costumbre de entregar por escrito datos personales para obtener un trabajo.

Existen datos que, en el año 1500, un peregrino inglés adjudicó su Currículo Vitae, otorgó este documento escrito a mano que contenía algunos datos personales.

Con el invento de la imprenta comenzaron a redactar modelos de currículos sobrepuestos una fotografía en blanco y negro, para entregar a las fábricas y otros lugares para poder emplearse.

A partir de 1960 empezaron a surgir formalmente los primeros currículos en la sociedad como instrumento importante para buscar empleo y desde entonces a progresado notablemente, actualmente este documento se ha convertido en un instrumento importante para conseguir empleo.

\section{Ventajas del Currículo Vitae}

Uno de los puntos más fuertes del currículo vitae es, que en él se presenta una opción perfecta para demonstrar sus conocimientos y experiencias en una oferta de trabajo.

Esta forma de currículum es muy útil para los aspirantes de trabajos que se enfocan dentro de una misma línea profesional, competitiva que demostrará en dicho campo.

Si se cambia de trabajo o de especialidad este tipo de currículo ayuda al aspirante a demostrar todas sus habilidades y experiencias, y si el aspirante tiene una edad madura se centrará en los conocimientos que posee.

\section{Currículo vitae funcional}

Este tipo de currículo se centra en las habilidades y experiencias de las personas, en lugar de explicar un historial de trabajo, también distribuye la información por argumentos.

\footnotetext{
419 Vol. 5, núm. 2, abril 2019, pp. 416-440 Gladys J. Negrete-Chauca; Eleana I. Rugel-Jiménez; Alfonsina B. Hernández-Valencia; Viviana F. Pinos-Medrano
} 
Proporciona un conocimiento rápido de la información que tienen las personas sobre los conocimientos y sus experiencias en ámbitos determinados.

Este currículo se centra en las destrezas y habilidades más que en la experiencia laboral o educativa.

El currículo vitae es más utilizado por personas que cambian de trabajo o de carreras que tienen escritas en su historial de profesión, oficio o cargo. Utilizan también las personas que son nuevas en los diferentes trabajos y que tienen poca experiencia laboral, y al poner de relieve sus habilidades más que su experiencia se puede enfatizar hasta qué punto se está calificando para el tipo de empleo que se está buscando.

El currículo Vitae es una herramienta importante de comunicación que define y ratifica profesionalmente, y a la vez permite demostrar el interés por una oferta de trabajo y también se acopla a cada caso concreto.

El principal objetivo del currículo vitae es lograr alcanzar una entrevista de trabajo ya sea en empresas o negocios privados o públicos, porque cada vez los empleadores buscan nuevos perfiles y requisitos especializados. Este tipo de currículo debe ser redactado de una forma concreta, clara, debe causar una buena impresión y no se debe cometer ningún error para no descartar la candidatura.

Existen algunos tipos de currículo vitae como el cronológico que se ordena desde el primer empleo que ha desempeñado o también desde el mas antiguo, la característica es el orden que debe seguir el candidato.

El currículo inverso, enfatiza la experiencia del aspirante.

El de proyectos destaca, subraya los diversos proyectos, capacidades, aptitudes y competencias del aspirante, siempre que se relacionen con la oferta de empleo.

También en ciertos casos se puede hablar del currículo misceláneo o mixto en el que se ordenan variadas estructuras para lograr destacar aspectos importantes, es útil para personas que tienen varios años trabajando en un mismo sitio o empresa y han logrado alcanzar muchos objetivos.

\footnotetext{
\begin{tabular}{l|l}
420 & Vol. 5, núm. 2, abril 2019, pp. 416-440 \\
Gladys J. Negrete-Chauca; Eleana I. Rugel-Jiménez; Alfonsina B. Hernández-Valencia; Viviana F.
\end{tabular} Pinos-Medrano
} 
Ejemplo de currículo vitae

- Datos personales:

- Nombre y apellidos:

- Dirección:

- Localidad:

- Teléfono:

- Email:

- Fecha de nacimiento:

- Estado Civil:

Formación de estudios

- Fecha:

- Institución educativa:

- Titulación:

Experiencia profesional

- Fecha:

- Empresa:

- Puesto. (Actividades desarrolladas)

Datos complementarios

- Idiomas:

- Instrucciones informáticas:

- Carnet de conducir. (Vehículo propio, disponibilidad geográfica)

- Lugar y fecha.

\section{El currículo educativo.}

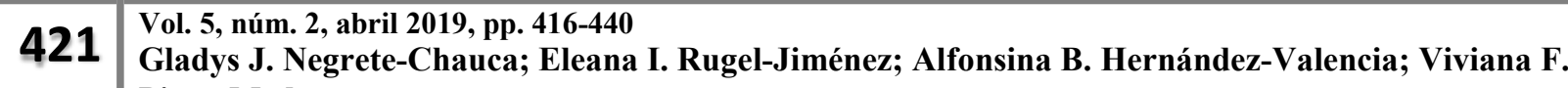
Pinos-Medrano
} 
"El currículo educativo es una serie de experiencias de entrenamiento, dirigidas a las escuelas, para completar y perfeccionar el desarrollo educativo para que los profesionales de la pedagogía empleen para ese objetivo" Franklin Bobbit (1918)

El currículo técnico, científico y especializado en educación se inicia a partir del siglo XX su primera publicación tuvo lugar en 1918 por Franklin Bobbitt, en su libro titulado The Curriculum. En este libro se constituyó la especialidad con modelos y ejemplos de programas educativos para todos los niveles de estudio.

Luego de la primera guerra mundial la mayoría de los países tuvieron un crecimiento industrial y un rápido proceso de urbanismo y desarrollo tecnológico. Atínea (2015) Manifiesta: "Estos hechos marcaron una influencia sobre la vida social, cultural, económica y política de América; así como también la producción de cambios dramáticos en los programas escolares: la comunidad demandaba de las escuelas programas que fueran utilitarios y efectivos"

La proyección de la organización y planificación del currículo tiene que seleccionar los objetivos trascendentales, significativos y alcanzables, porque el propósito de la educación es promover cambios importantes tanto en comportamiento como en el nivel de aprendizaje de los estudiantes. Se utilizará todas y cada una de las estrategias y actividades didácticas, para alcanzar los objetivos propuestos.

Entonces el currículo se compone por una serie de razonamientos, procedimientos de estudio, presentaciones, metodologías, técnicas, métodos, procesos que contribuyen a la formación general, sistémica y a la cimentación de la identidad e igualdad cultural local, regional y nacional, conteniendo los recursos humanos, académicos y físicos para orientar en práctica las capacidades, estrategias, habilidades y llevar a cabo el programa educativo institucional.

Se puede decir que el currículo educativo es la organización, ordenación y distribución importante de un contenido o asignatura dentro de cualquier institución educativa, como puede ser para educación general básica, media y superior.

\footnotetext{
422 Vol. 5, núm. 2, abril 2019, pp. 416-440 Gladys J. Negrete-Chauca; Eleana I. Rugel-Jiménez; Alfonsina B. Hernández-Valencia; Viviana F. Pinos-Medrano
} 
Luego de la publicación en el año de 1999 de un escrito de Herbst Jurgen y en el 2001 de las posteriores copias al mismo, la Revista Pedagógica se convierte en el foco del debate de varios pedagogos sobre la leyenda del presente, pasado y futuro del currículo educativo.

Estos continuos debates generaron muchas discrepancias de interpretación y de valor sobre su metodología y aplicación. Temas que no se resuelven, sino que precisan la permanente revisión y valoración de la reflexión crítica y autocrítica.

Es conocido por todos que las propuestas de mejoras no deben estar desprovistas de la realidad, como menciona Lerena Carlos, la historia no es solo eso que pasa, sino también eso que pesa sobre las instituciones educativas, los comportamientos y las conciencias de todos los responsables, condicionando las propuestas y posibilidades de cambio.

Tyack- Tibin (1949) han denominado como la "gramática básica de la instrucción" por ello la posibilidad se hace difícil por el distanciamiento que se presenta como que lo familiar parezca raro y lo natural ilegal, esto sin mirar la propia socialización en las normas no escritas que dirigen la cultura escolar.

Bourdieu (1999: 23) manifiesta: "Solo la ilusión de la omnipotencia del pensamiento puede hacer creer que la duda más radical tenga la virtud de dejar en suspenso los presupuestos, relacionados con las diferentes filiaciones, pertenencias, implicaciones que fluyen en los pensamientos" Lo instintivo es la historia que ha derivado categorías de pensamiento, y mediante la leyenda individual, por medio de la cual han sido inculcadas a las diferentes generaciones.

Desde las propias inquietudes son muchos los motivos que promueven a remover en la historia de los regímenes escolares, de sus clasificaciones gramaticales, de los comportamientos de la educación, de la dirección de infantes, niños, adolescentes, para mantener una crítica profesional, psicoanalítica; para los estudiantes y docentes en la actualidad.

Buscar en la historia pistas para entender a los estudiantes actuales a los que se destinan las propuestas de las diferentes asignaturas y distintos funcionarios que han configurado las innovaciones, tradiciones, mitos, ritos que rigen determinados criterios de organización y selección de los variados contenidos de estudio.

\footnotetext{
423 Vol. 5, núm. 2, abril 2019, pp. 416-440 Gladys J. Negrete-Chauca; Eleana I. Rugel-Jiménez; Alfonsina B. Hernández-Valencia; Viviana F. Pinos-Medrano
} 
Al responsabilizarse de la enseñanza y del aprendizaje de una parte del currículo, no solamente se toma el curso de estudios sino también todos los contenidos de la escolaridad, tomando en cuenta la planificación de la mediación didáctica.

El currículo englobado será llevado a cabo en las interacciones entre: directivos, estudiantes, docentes y toda la comunidad educativa, a través de acomodaciones, interpretaciones, negociaciones, controles, interacciones, actuaciones, resistencias, omisiones, rechazos que lo reforman en el espacio físico e institucional, ajustando a las aulas el paradigma o modelo de experiencias educativas.

Young (1998) Ha subdividido en dos variantes denominadas currículo como hecho y currículo como práctica.

- El currículo como hecho, una perspectiva que presenta expresamente el conocimiento a impartir en los centros de estudio como una forma dada y configurada anticipadamente todo el conocimiento a impartir en las escuelas, colegios y universidades como un objeto dado.

- El aprendizaje consiste entonces en una iniciación del conocimiento a través de las asignaturas, porque las excelencias culturales son estructuradas epistemológicamente objetivas, irreductibles, puesto que el trabajo de las instituciones educativas es transmitir conocimientos dispuestos en el currículo, mediante procesos disciplinares.

- El currículo como práctica, se localiza en las estructuras del conocimiento, separadas de los sujetos, por sucesos que ocurren en el aula, el beneficio de las prácticas de los docentes y de los estudiantes en el contorno de los eventos singulares que rodean las interacciones y transacciones cotidianas, como la enseñanza, el aprendizaje, las diferencias individuales, los saberes valiosos, la autoridad, los patrones de conducta, el orden, la transgresión, la resistencia, los aciertos y desaciertos, las consecuencias de los diversos actos, entre otros.

El currículo efectivo no es un objeto prefabricado, impartido o recibido por los receptores en los lugares de su aplicación, sino que es conformado por los protagonistas, a través de las acciones que lo dotan de sentido.

Lo que configura al currículo no son las preocupaciones y acciones estrictamente de carácter formativo cognitivo, sino también las estrategias de duración, de adaptación de unos y otros,

\footnotetext{
424 Vol. 5, núm. 2, abril 2019, pp. 416-440 Gladys J. Negrete-Chauca; Eleana I. Rugel-Jiménez; Alfonsina B. Hernández-Valencia; Viviana F. Pinos-Medrano
} 
mediante las respectivas culturas, hábitos, creencias, normas, experiencias y posibilidades. Por esta razón al conocimiento ya no se trata como propiedad privada, sino como la interacción entre docentes y estudiantes.

El currículo comenzó a tener más énfasis a finales de la década de los 70 luego de la revuelta de algunos grupos universitarios frente al pensamiento dominante de los organismos encargados de las reformas, que distanciaban a los estudiantes de los docentes.

Desde el punto de vista del currículo como práctica ha cuestionado los pensamientos en lo cultural, restituyendo a docentes y estudiantes a la dignidad de personas activas, para la organización de la educación. Todo currículo se edifica sobre la base de las transacciones e interacciones de los estudiantes en el aula de clase, mediante la socialización de contenidos, reglas, normas que rigen su definición.

\section{El currículo y su alcance.}

Desde mucho tiempo la educación se ha planteado muchos desafíos, el principal es llevar con éxito el proceso de enseñanza aprendizaje, mediante la principal herramienta que es el currículo educativo, porque permite diseñar aspectos importantes, como estrategias de enseñanza, procedimientos coherentes, entre otros.

El currículo no solo es la respuesta de lo que quieren lograr las instituciones educativas, sino que ayuda a crear un clima agradable, propicio, flexible, que permita a los docentes y estudiantes desarrollar el pensamiento creativo, para lograr los objetivos propuestos.

Esta herramienta ayudará a fortalecer los procesos de la enseñanza y del aprendizaje, mediante la construcción del conocimiento, utilizando todos los instrumentos necesarios, para aplicar en el aula de clase y evaluar su desempeño.

En todo periodo de enseñanza el currículo es la guía para prever, ordenar, establecer, comprobar y gestionar que los procesos de enseñanza y aprendizaje se esté realizando de forma satisfactoria tanto para estudiante, docentes y comunidad educativa.

\footnotetext{
425 Vol. 5, núm. 2, abril 2019, pp. 416-440 Gladys J. Negrete-Chauca; Eleana I. Rugel-Jiménez; Alfonsina B. Hernández-Valencia; Viviana F. Pinos-Medrano
} 
Es importante la ayuda del currículo en la práctica docente porque beneficia al maestro a manejar el papel de guía de la enseñanza dentro y fuera del aula de clases, que permita a los estudiantes progresar de forma general, holístico e integral que envuelva todos los aspectos importantes de su progreso.

\section{Importancia del currículo en la educación}

- Lograr que los estudiantes obtengan un elevado nivel de conocimientos, habilidades y actitudes correctas, para poder desarrollarse adecuadamente en el diario vivir.

- Mejorar la enseñanza tomando en cuenta la individualidad de cada estudiante.

- Estar al tanto de los objetivos y fundamentos teóricos para realizar el proceso de la enseñanza y el aprendizaje.

- Educar con responsabilidad para responder a todas las necesidades, económicas, sociales y políticas de un país.

- El currículo contribuye a formar estudiantes en el ámbito educativo, social, económico, político, en un ambiente libre, lúdico y afectivo.

- $\quad$ Fortalecer el proceso de la enseñanza y el aprendizaje.

Entre los aspectos más importantes del currículo se encuentran:

- Construir.

- Instrumentar.

- Aplicar.

- Evaluar.

Estos aspectos son trascendentales para su esencial relevancia que es comprobar, guiar, prever, organizar, gestionar que el aprendizaje sea agradable, grato y placentero, tanto para estudiantes como para docentes que son guía de la enseñanza dentro y fuera del aula de clase.

Por lo tanto, el currículo está centrado en formar estudiantes, docentes y comunidad educativa, con saberes significativos, específicos adecuados a los avances tecnológicos y a los cambios constantes del mundo moderno. El currículo permite formar personas de distintas características, habilidades, sentimientos, defectos, virtudes.

\footnotetext{
426 Vol. 5, núm. 2, abril 2019, pp. 416-440
Gladys J. Negrete-Chauca; Eleana I. Rugel-Jiménez; Alfonsina B. Hernández-Valencia; Viviana F. Pinos-Medrano
} 
Los elementos básicos del currículo son los objetivos que describen, relatan, representan el propósito del contenido de una o varias asignaturas que precisan, delimitan qué se quiere lograr con el aprendizaje del currículo educativo y cuál será la ordenación, alineación, disposición y distribución principal del docente, cuyos contenidos abarcan y comprenden, conceptos teóricos, prácticos, procedimentales, metodológicos y estratégicos.

La metodología comprende las maneras en que las diversas asignaturas serán abordadas y los objetivos justos e imparciales se alcanzarán con el resultado del nivel de aprendizaje.

Es útil presentar un currículo educativo con objetivos precisos, puntuales, formales y riguroso, con contenidos claros, despejados, metodologías coherentes y relacionadas con las asignaturas y un formato de valoración que retroalimente el conocimiento adquirido, para que los estudiantes interioricen con más facilidad los nuevos conocimientos.

\section{Teorías del currículo.}

"El origen del currículo en el campo de la investigación es la inquietud social, política de tratar de resolver las necesidades y problemas educativos de un país” López 2002.

Lo que se proyecta con las diversas teorías y estudios del currículo es para proporcionar un procedimiento fundamentado y sistemático a las decisiones de los pedagogos de los diferentes países, sobre cómo y qué enseñar a los estudiantes en general, dándole sentido a la educación para poder alcanzar los objetivos establecidos con anterioridad.

Existen algunos autores que estudiaron el currículo educativo con sus concepciones respectivas, en la actualidad ya no se usan estos procesos metodológicos, porque toda la sociedad está inmersa en un constante cambio e innovación, pero para poder crear o innovar se tiene que tener conocimiento de su origen y desarrollo.

Para diseñar un currículo es necesario planificar cada una de sus partes minuciosamente para comprender lo que es y lo que se va hacer, porque está en juego la idea, el proyecto, la concepción de sociedad y estudiantes y están supeditados a un sistema de evaluación.

\footnotetext{
427 Vol. 5, núm. 2, abril 2019, pp. 416-440 Gladys J. Negrete-Chauca; Eleana I. Rugel-Jiménez; Alfonsina B. Hernández-Valencia; Viviana F. Pinos-Medrano
} 
También se pronunciará sobre la reciprocidad que la valoración e innovación que establecen con la sociedad, estos planes se hacen sobre valores, experiencia, cultura, libertad, el pasado, presente y el futuro de todos los estudiantes en general.

Es importante hacer una breve reflexión sobre las diferentes teorías curriculares para poder hacer una crítica constructiva de cada una de ellas y tomar la mejor opción.

Kemmis (1988) Dice: "El problema interno de la teoría del currículo debe ser entendido como el doble problema de las relaciones entre la teoría y la práctica por un lado y de las relaciones entre la sociedad y la educación"

Estas recomendaciones han sido demostradas, explicadas y planteadas de distintos modos a lo largo de la historia, este planteamiento designado por la sociedad para representar la organización y estructura del conocimiento, deben reflejar los valores éticos, morales y tradiciones de toda la sociedad, en aplicación y función del papel que posee la educación y su relación con el currículo educativo en el trato práctico de la vida y en la responsabilidad del trabajo de las personas.

Desde hace décadas las diversas teorías han evolucionado a la par de la tecnología, tanto en la forma de entender el establecimiento social, cultural, político y hasta religioso de la educación.

Para Kemmis la teoría técnica del currículo razona, imagina, medita sobre la sociedad, la ciencia, el conocimiento y la cultura como una conspiración externa al discipulado y al currículo como un contenido caracterizado por las necesidades y los objetivos generales a los que la educación está obligada a responder, desarrollando programas adecuados tecnológicamente para poder alcanzar los objetivos de las grandes mayorías.

En cambio, la teoría práctica del currículo imagina a la sociedad como un tipo de fundamento, más activo sobre el papel de educación de las diferentes instituciones educativas, de los docentes y su contribución con la sociedad, logrando tener individuos con pensamientos críticos y creativos.

En cuanto a la teoría crítica del currículo Kemmis manifiesta: "El currículo es parte de la premisa que las estructuras sociales no son tan racionales y justas como generalmente se piensa"

\footnotetext{
428 Vol. 5, núm. 2, abril 2019, pp. 416-440 Gladys J. Negrete-Chauca; Eleana I. Rugel-Jiménez; Alfonsina B. Hernández-Valencia; Viviana F. Pinos-Medrano
} 
En la segunda década del siglo XX se inicia la teoría crítica como resistencia a los planteamientos positivistas, autoritarios que dominaban a las ciencias exclusivamente relacionando a la producción de bienes y servicios.

Según Kemmis se puede pensar que las organizaciones sociales se han creado mediante técnicas, métodos y hasta conocimientos distorsionados, deformados, por la irracionalidad, el despropósito, la injusticia, la restricción y han atravesado profundamente las explicaciones e interpretaciones del mundo.

El conocimiento de estos procesos revelan algunas maneras distorsionadas en la vida social y puntos de vista negativos sobre ella, para evitar todos estos procesos y puntos de vista negativos es necesario elaborar el currículo adecuadamente, flexible, pensando en cada tipos de sociedad que tiene cada país.

Horkheimer (1930) Expresa: "El papel de la ciencia se convertía en técnico al alimentar el razonamiento instrumental y proporcionar los métodos y principios para resolver los problemas técnicos de la producción"

En aquella época se creía que la ciencia tenia el poder para reconocer a todas las preguntas y cuestiones significativas, los hipotéticos críticos veían peligroso para la sociedad moderna porque solo importaba remplazar con la técnica para avanzar en la producción de infinidad de artículos, mientras que el currículo no estaba sometido a revisión ni cumplía con los objetivos que puedan justificar las líneas de acción para la cual estaba previsto.

Para avanzar en el proyecto tecnológico de educación y la orientación racional para desarrollar, planificar, implementar y valorar el currículo la lógica instrumental satura la conciencia de los docentes, de las escuelas, colegios y universidades e interviene utilizando el idioma de la ciencia de la eficacia y valides de la empresa, de la utilidad y del dinero.

Si la educación quiere avanzar y cumplir con sus cometidos se debe proyectar en términos de racionalidad científica para no quedar separada, desvinculada, aislada de la modernidad y de los enfoques sistémicos de la informática. El currículo educativo debe estar relacionado con los objetivos, con sus evaluaciones, entradas, salidas y no permitir que quede nada imprevisto.

\footnotetext{
429 Vol. 5, núm. 2, abril 2019, pp. 416-440
Gladys J. Negrete-Chauca; Eleana I. Rugel-Jiménez; Alfonsina B. Hernández-Valencia; Viviana F. Pinos-Medrano
} 
En cuanto al enfoque de la eficiencia social establece que el currículo será desarrollado de una forma científica porque su desarrollo adecuado es para satisfacer las necesidades de la sociedad.

La esencia de todo ser humano se encuentra en las capacidades y habilidades que es capaz de realizar y eso se obtiene a través de la educación, por este motivo los desarrolladores de los currículos deben establecer las distintas necesidades de la sociedad.

El enfoque académico admite que existe una equivalencia entre las asignaturas académicas en el mundo del entendimiento y del conocimiento, esto se logrará mediante la integración de los estudiantes, a través de la transmisión de culturas, ciencias e instrucciones.

El currículo centrado en el estudiante focaliza los intereses directamente sobre el niño, joven y adulto, se debe crear un currículo conforme con la naturaleza innata del ser humano, para cubrir las necesidades e intereses individuales.

Este tipo de currículo concibe al estudiante como un ser capaz de su propio desarrollo, como actor que debe modificar sus propias capacidades y fundamentalmente humano y bueno por naturaleza, además el niño es visto como una inagotable fuente de contenidos para el currículo.

El objetivo central del currículo es el crecimiento y desenvolvimiento del estudiante acorde a las leyes de su existencia, produciéndose en el niño, joven y adulto aprendizajes sanos, beneficiosos $\mathrm{y}$ virtuosos porque responde a las capacidades innatas del ser humano.

Los expertos en desarrollar el currículo creen que la sociedad, el ser humano y la educación están sufriendo una tremenda crisis y el objetivo de desarrollar el currículo es para modificar esta realidad y tratar de eliminar de su cultura aquellos aspectos negativos y sustituir por todos los valores para que la sociedad logre conseguir la satisfacción de todas las necesidades sean estas materiales y espirituales. Para lograr obtener un currículo que sea capaz de alcanzar todos los objetivos propuestos es necesario redactarlo mediante un consenso social que evite errores y que admita las virtudes de la futura sociedad.

John Dewey (1859-1952) Expresa: "El currículo como favorecedor de las necesidades de los niños por sobre las necesidades sociales, desarrollando un currículo de alto contenido práctico"

\footnotetext{
430 Vol. 5, núm. 2, abril 2019, pp. 416-440 Gladys J. Negrete-Chauca; Eleana I. Rugel-Jiménez; Alfonsina B. Hernández-Valencia; Viviana F. Pinos-Medrano
} 
Dewey en 1949 retorna el pensamiento fundamentalista del currículo educativo, con contenidos creados por expertos.

En 1967 Robert Gagné, define el currículum como una secuencia de complejidad creciente, donde las nuevas capacidades, cuenten con otras anteriormente adquiridas.

La pedagoga Alicia de Alba, (1991) Dice: "Que el currículum sintetiza diversos elementos culturales, entre ellos, conocimientos, creencias y valores, donde se concretan a través de la negociación o la imposición, las ideas e intereses de diversos sectores, para conformar una propuesta educativa, que es también una propuesta política"

En los años de 1990 el problema en cuanto al currículo es mostrar cual es el conjunto de decisiones, generales que debe componer junto a su fundamento, para establecer una propuesta de enseñanza sólida, resistente y realizable. Las fallas que han presentado las diferentes teorías curriculares se han dado por la falta de análisis de varios problemas como la disposición de lo que se debería enseñar o lo que los estudiantes deben aprender.

Las decisiones tomadas sin la mayor responsabilidad de quienes participan en la estructura del currículo, o porque el currículo real no es idéntico al presentado por la autoridad educativa.

Schwab. "Argumenta favor de la práctica, en un intento de resucitar el antiguo tipo de teoría educativa, centrándose especialmente en la toma práctica de decisiones de los profesores en las clases y en las comisiones del currículum de la escuela"

En cuanto a la aplicación de la práctica no debe limitarse a la técnica, sino referirse a un marco teórico que contemple la naturaleza de los fines perseguidos.

La teoría de la interdisciplinariedad presenta varias ideas y diferentes opciones para el análisis y la enunciación de propuestas y permite establecer teorías que pueden desembocar varios niveles no muy positivos porque el currículo es un cuerpo teórico de frágil integración.

El currículo condiciona el trabajo de los profesores en tanto accede un marco de acción, porque no solo emprende u organiza contenidos, espacio, tiempo, metodología, sino encuadra el modo de pensar.

\footnotetext{
431 Vol. 5, núm. 2, abril 2019, pp. 416-440

Gladys J. Negrete-Chauca; Eleana I. Rugel-Jiménez; Alfonsina B. Hernández-Valencia; Viviana F. Pinos-Medrano
} 
Bernstein Propone algunas formas de reglamentación del trabajo docente, como el control simbólico originando determinados valores, métodos, ciencias, pensamientos justificando una disertación frente a otra. El control instrumental establece técnicas, codificaciones, procedimientos y tecnologías. También estimula la libertad crítica y genera reflexión sobre los determinantes del conector escolar y docencia, en la trayectoria a la construcción de una nueva influencia en las disposiciones escolares.

Bernstein entiende al currículo como un conector pedagógico organizado directamente en función de principios.

Como manifiesta Bernstein sobre las comunidades de clase media que buscan para sus hijos escuelas con diferentes maneras de formación pedagógica, según su grado de escolaridad.

Con el paso del tiempo el currículo evaluado por la enseñanza inicial, media y superior, por la calidad de la educación académica y disciplinaria mas intensa, ya que las instituciones educativas extienden su enseñanza de forma interdisciplinaria.

En las teorías experienciales del currículo el pedagogo Dewey postuló una pedagogía centrada en la formación de los estudiantes para la democracia y la libertad, formada como una sociedad razonada, legítima, progresista, liberal y humanista, pero no estaba muy centrada en las necesidades de los estudiantes como en la organización para la democracia.

El enfoque del currículo centrado en los estudiantes no se refiere solamente al procedimiento o programa pedagógico, sino que contiene formas alternativas de organización escolar dentro de las áreas o espacios, materiales didácticos, muebles escolares y tiempos.

De esta manera el currículo experiencial concibe, razona, alcanza a todas las prácticas de aprendizaje que proporciona la institución educativa o el conjunto de acciones planificadas por la escuela para ayudar a los estudiantes alcanzar un alto nivel los objetivos prescritos.

Los autores del currículo centrados en las concepciones liberales acuerdan dar a la mayoría la oportunidad de experiencias educativas, demostrando diferencias sociales y logros en varias capacidades individuales, en consecuencia, no muestran explicaciones que permitan alcanzar,

\footnotetext{
432 Vol. 5, núm. 2, abril 2019, pp. 416-440 Gladys J. Negrete-Chauca; Eleana I. Rugel-Jiménez; Alfonsina B. Hernández-Valencia; Viviana F. Pinos-Medrano
} 
entender resultados diferenciales en el adiestramiento y aprendizaje de acuerdo a niveles socioeconómicos y todo tipo de minorías comunitarias.

En lo que respecta a las teorías formativas del currículo, la perspectiva o disposición de la ciencia aplicada se convierte en hegemónica a partir del siglo XX. La ciencia empieza a concebir como la clave del adelanto o progreso y esta idea o concepción domina el pensamiento pedagógico.

La base del cambio social es el conocimiento considerado como fruto del desarrollo científico y la misión de las instituciones educativas es intercambiar y distribuir el conocimiento masivamente, lo que requiere de la ayuda estatal y de organismos internacionales.

La confección del currículo debe estar a la altura de las circunstancias, realidades o contextos, que aspira a reconocer y responder a las demandas de la sociedad, porque se recurrió a los conocimientos especializados de distintas ciencias y sus múltiples investigaciones empíricas.

La división del trabajo en el ámbito educativo introducido por las nuevas teorías curriculares, hizo que los especialistas del currículo se mantengan apartados, por un lado los docentes, por el otro a estudiantes. Esta orientación generó una serie de herramientas para establecer objetivos escolares y resultados técnicos específicos para construir el currículo.

Tyler resalta secuenciaciones técnicas similares para construir el currículo, las dificultades teóricas a estudiar y a resolver en la práctica son varias por las múltiples interrelaciones, la causa o el proceso educativo reside en el dominio del contenido y en el desarrollo de las diferentes facultades.

Los docentes son los responsables de facilitar los procesos de enseñanza y del aprendizaje, dinamizando la diligencia de los alumnos, los padres y madres de famiia y los miembros de la comunidad educativa.

También permite al niño y joven conocer sus capacidades y limitaciones, edificar un perfil realista de si mismo, diseñar metas viables para desplegar sus capacidades afectivas, cognitivas y sociales.

\footnotetext{
433 Vol. 5, núm. 2, abril 2019, pp. 416-440 Gladys J. Negrete-Chauca; Eleana I. Rugel-Jiménez; Alfonsina B. Hernández-Valencia; Viviana F. Pinos-Medrano
} 
En definitiva las teorías y las prácticas pedagógicas se fundamentan por normas, métodos humanísticos como son la filosofía, epistemología de la educación, la psicología, la antropología, la sociología, en cada uno de los periodos históricos de la humanidad han ido madurando, considerando sobre la idea, el pensamiento del ser humano, su razón de ser. En esta representación el currículo nace como la guía para educar y perfeccionar a estudiantes sean estos niños, jóvenes o adultos en sus relaciones con la sociedad.

El currículo educativo ha evolucionado a través de la historia, en la concepción, conocimiento y percepción de instrucción y de enseñanza y aprendizaje.

Miel, A. (1976) Manifiesta que currículo no es un programa de estudio sino las experiencias que los niños logran bajo las orientaciones de la escuela. Lo que da a entender que el currículo ayuda a descubrir puntos de aprendizaje planeados y guiados por las instituciones educativas.

Bloon, M. (1994) Dice: "El curriculo es un espacio de saber en donde se inscriben disertaciones, procedimientos y técnicas para la organización, diseño, programación, planificación y administración de la educación, bajo el principio de la determinación previa de objetivos específicos planteados en términos de comportamientos, habilidades y destrezas con el propósito central de obtener un aprendizaje efectivo"

Como lo manifiesta Bloon el currículo es un conjunto ordenado de normas, reglas, políticas, criterios, principios que orientan el proceso educativo, mediante la enunciación de objetivos, metodologías, logros, criterios de evaluación y desempeños.

\section{Senda histórica del curriculo.}

Abraham Magendzo describe varios ejemplos importantes sobre la historia de la humanidad que se presenta a continuación: Con respecto a a República de Platón, luego Aristételes la educación estaba jerarquizada de acuerdo a las inclinaciones y deseos, el temple, la importancia del espíritu, el intelecto y la razón. Esta diferencia del currículo servía para formar a unas personas en arte, para el trabajo y a otras para las ciencias.

\footnotetext{
434 Vol. 5, núm. 2, abril 2019, pp. 416-440

Gladys J. Negrete-Chauca; Eleana I. Rugel-Jiménez; Alfonsina B. Hernández-Valencia; Viviana F. Pinos-Medrano
} 
En los siglos XVI y XVII, con el avance científico, político y social, poco a poco la educación se va separando de las bases escolásticas y comenzó a progresar hacia la construcción de pensadores y pdagógos.

Rousseau hace que la educación alumbre como parte general de las reformas y transformación social. Para Dewey, la educación es un medio y un fin para edificar la democracia. El manifiesta que el objetivo principal es la formación de hábitos, experiencias y costumbrees, modos de ser, reflexiones, ideologías, pensamientos y sentimientos propios de las sociedades. Tambien dice que la democracia y la educación se relacionan directamente y serán consideradas desde las prácticas pedagógicas.

En Latinoamérica en los años cuarenta se dice que la educación es el medio para la transformación social, que exige que los países se responsabilicen con el derecho que tiene cada ciudadano a la educación.

A finales de la década de los años cincuenta se inicia un progreso de divulgación de la educación primaria que debía ser obligatoria y gratuita en todos los países en desarrollo. La educación tendrá su cimiento para la expansión social y la ejecución de planes y programas de estudio, eleborados de forma sistemática, ejerciendo un conjunto dde técnicas en la planificación.

El curriculo es un experimento nuevo en la historia educativa y aparece atada a las disertaciones y prácticas sobre la planificación y el perfeccionamiento, provenientes de los países en desarrollo.

En la década de 1960 y 1970 la proyección general y la tecnología instruccional cobran vigencia, porque el diseño general fue el dispositivo, para lograr las metas del desarrollo mediante la educación, con un proceso continuo y sistemático en el que se coordinan y se aplican métodos, tecnicas y conocimientos de investigación social.

La tecnología estuvo especializada en, análisis de tareas, evaluación de procesos, transformación de comportamientos y valoración de resultados. En la década de los de los años 80 , tuvo lugar la revolución curricular junto con la tecnología dieron paso a la organización del sistema educativo en muchos países.

\footnotetext{
435 Vol. 5, núm. 2, abril 2019, pp. 416-440
Gladys J. Negrete-Chauca; Eleana I. Rugel-Jiménez; Alfonsina B. Hernández-Valencia; Viviana F. Pinos-Medrano
} 
La tecnología educativa se presenta como un procedimiento sistemático y basado en análisis y solución de problemas en las instituciones educativas de todos los niveles. Esta tecnología se convierte en instrumento importante y eficaz para establecer, instaurar y fundar los objetivos que debe desarrollar la reforma de la educación.

Las dificultades educativas son muy complejas porque son concernientes a docentes, a los procesos de la enseñanza y el aprendizaje mediante el uso de variadas metodologías, técnicas, estrategias y ordenamientos o programaciones.

En 1990 comienzan los procesos globalizadores en todos los campos educativos y empieza el nuevo proceso de desarrollo curricular basado en competencias. Estas competencias permiten comprender, argumentar, demostrar, resolver dificultades ambientales, sociales y tecnológicos.

Como conclusión todas las teorías curriculares son modelos, el docente debería tener la libertad de elegir la que mejor crea conveniente y se ajuste al contexto y de acuerdo a su personalidad, al grupo de estudiantes para ejecutar de manera adecuada cada día en el aula de clase, tomando muy en cuenta el sublime fin de la educación el cual es preparar a los niños y jóvenes para la vida en sociedad y engrandecimiento del país y el mundo.

\section{El currículo oculto.}

Todos los aprendizajes se que se concentran por los estudiantes se llama currículo oculto, aunque muchos aspectos no formen parte del currículo oficial. Según los contextos, situaciones, condiciones y dichos contenidos no pueden ser enseñados. Cualquier actividad social, política, administrativa, religiosa, doméstica brinda aprendizajes no buscados, porque el aprendizaje no se vincula a los centros educativos, sino a las experiencias que día a día tienen que sortear los seres humanos.

Continuamente el currículo oculto se describe o se refiere a conocimientos, ciencias e instrucciones adquiridas fuera del aula de clase, es decir solamente mediante la experiencia vivida día tras día a lo largo de la existencia.

\footnotetext{
436 Vol. 5, núm. 2, abril 2019, pp. 416-440 Gladys J. Negrete-Chauca; Eleana I. Rugel-Jiménez; Alfonsina B. Hernández-Valencia; Viviana F. Pinos-Medrano
} 
En el mundo actual los valores de las personas han ido disminuyendo con el pasar de los días, por este motivo los sistemas educativos no reconocen ante lo legal ese tipo de conocimientos o instrucción.

El currículo oculto también interioriza normas, creencias, valores, interacciones sociales, cimientos políticos, religiosos, culturales, entre otras. Estas faenas fueron influenciados, afectados por los grupos de explotadores que con el tiempo se ha perdido el interés y la importancia.

Desde el punto de vista estructural el currículo oculto interioriza los valores y normas en las personas producto de la experiencia y del día a día durante el pasar de los años. Desde el punto de vista fenomenológico este currículo es creado a través de interacciones, esto implica que el conocimiento es objetivo.

Algunos aspectos del aprendizaje contribuyen a la amplificación del currículo oculto como la autoridad, la estructura social, los libros y demás materiales para la autoeducación, varias medidas disciplinarias, también se transmiten conocimiento con gestos y movimientos corporales.

Este tipo de currículo es fácil de detectar, pero en muchas ocasiones es muy discriminado. Entre los materiales e instrumentos que usa el currículo oculto se encuentran libros, enciclopedias, conocimientos de los individuos del entorno, experiencias de todo nivel cultural de las personas. Todos estos conocimientos que las personas y los instrumentos de educación brindan se convierten en una influencia importante en su formación de niños, jóvenes y adultos, porque se transmite enseñanzas y aprendizajes, didácticas, morales, sociales y comportamentales.

El currículo oculto incorpora variedad de conocimientos a niños, jóvenes y adultos en pocas ocasiones tiene una relación o connotación negativa, por los valores que se puede promover debido a diferentes estratos sociales como lo afirma Bourdieu Pierre, que dice: "Que el capital relacionado con la instrucción o educación debe ser accesible para promover los logros académicos" Pierre esta convencido que el currículo oculto constituye una forma básica educativa y que su eficiencia muchas veces depende de la orientación y capacidad que poseen las personas que interiorizan conocimientos.

\footnotetext{
437 Vol. 5, núm. 2, abril 2019, pp. 416-440 Gladys J. Negrete-Chauca; Eleana I. Rugel-Jiménez; Alfonsina B. Hernández-Valencia; Viviana F. Pinos-Medrano
} 
Según Elizabeth Vallance, las funciones del currículo oculto, la interiorización de valores, el adiestramiento en la obediencia, la socialización de las estrategias, la perpetuación de las funciones estructurales de las clases tradicionales puede ser caracterizadas generalmente como control social.

También el currículo oculto puede estar mancomunado con el reforzamiento de la desigualdad social, como se ha visto por el desarrollo de las diversas relaciones básicas de los prototipos de trabajos y actividades relacionadas con personas que se encuentran auto educándose de diferentes clases sociales.

Los estudios del currículo oculto se ajustan a los estudiantes de niveles de primaria y secundaria y los estudios superiores se ven afectados por los efectos de estos conocimientos, por la calidad y las experiencias asociadas a la instrucción, este precedente se hace mas significativo muchas veces en el género y raza.

Este procedimiento de imponer trayectos educacionales entre estudiantes (niños y adolecentes) se apoyan en varios factores como son la clase y el estatus social se muestra mas flexible para superar las diferencias socioeconómicas.

\section{Reseñas intelectuales.}

John Dewey investigó el currículo oculto del aprendizaje en los trabajos a inicios del siglo $\mathrm{XX}$, en exclusivo en su clásico Democracia y educación y encontró progresos en los patrones y desarrollos de tendencias en las instituciones educativas públicas que se prestaban a sus perspectivas de avanzar diciendo que los estudiantes de todas las edades se convertirán en personas creativas y multifacéticas.

George Counts en la obra de 1929: "Reto a la escuela a construir un nuevo orden social" comprobó, examino la naturaleza de las responsabilidades de Dewey, mientras que otros pedagogos lanzaron hipótesis sobre un recorrido único que tanto niños como jóvenes caminan hacia el futuro para convertirse en adultos.

Counts determino la naturaleza del aprendizaje como adaptativa, reactiva y multifacética, interdisciplinaria, pluridisciplinarias y multidisciplinaria. El concepto de currículo oculto fue

\footnotetext{
438 Vol. 5, núm. 2, abril 2019, pp. 416-440 Gladys J. Negrete-Chauca; Eleana I. Rugel-Jiménez; Alfonsina B. Hernández-Valencia; Viviana F. Pinos-Medrano
} 
contrastado por Jackson Philip en 1968 en su escrito "Vida en la clase" donde se certifica que la educación es un proceso de socialización.

Meughan en su obra "Una sociología de la educación en 1981 decía que el currículo oculto es enseñado en la escuela no por docentes sino por la experiencia de cada uno de los estudiantes que llega a convertirse en una perspectiva de vida y una forma de aprendizaje.

Para Haralambos Michael (1991) manifiesta en su obra Temas y perspectivas, que el currículo oculto radica en aquellas formas que los estudiantes aprenden a través de la experiencia de las personas en su diario vivir.

En el currículo oculto el proceso de enseñanza aprendizaje o inter-aprendizaje, puede salir afectado por el insuficiente conocimiento que adquieren los estudiantes por la escasa metodología y bases pedagógicas de educación.

Aspectos significativos del diseño curricular:

- Permite al estudiante conocer sus contenidos y especificaciones, plantearse metas viables para ampliar las capacidades cognitivas, afectivas, expresivas, precisas, formales y sociales.

- En cuanto a la valoración debe tener carácter general, sistémico y social.

- El desarrollo de programas para mejorar el nivel de enseñanza y aprendizaje.

- La interacción estudiante - docente es reconocida como una parte importante del currículo oculto progresivo.

- Inclusión de los estudiantes en los centros educativos

- Poder comprender lo complejo del proceso de enseñanza aprendizaje y la calidad de la formación integral de los futuros profesionales.

La metodología del currículo establece con claridad para los niños y jóvenes que asisten a las instituciones educativas, que se interesa por el cumplimiento de objetivos, propósitos, ideales, claves y metas de la educación.

Para el currículo el principal objetivo es la integración de la diversidad de los estudiantes.

\section{Conclusiones.}


Como conclusión el currículo es un orden de contenidos, conocimientos, culturas, sapiencias y experiencias vividas para lograr objetivos de enseñanza, aprendizaje y guía para el docente y demás personas que se sirven de este valioso instrumento, que promueve el desarrollo humano a partir de su fortaleza, ánimo, empuje, voluntad y esfuerzo que tiene su contenido.

En definitiva, si se pretende integrar estudiantes en el respeto, el compañerismo, el aprendizaje, la afectividad, la solidaridad y demás valores se debe acudir al currículo que debería ser ciento por ciento flexible.

\section{Bibliografía.}

Davini, M. (1999) Enfoques teóricos del currículo.

Gimeno, S. (1986) Obsesión por la excelencia. Madrid.

Kemmis, S. (1988) Hacia la teoría critica del currículo. Madrid.

Casarini, R. (2010) Teoría y diseño curricular. Trillas.

Dinerstein, P. (2009) Diseño curricular. Argentina.

Drummond, S. (2011) Metodología de diseño curricular.

Cuesta, R. (1997) Disciplina escolar. Barcelona.

Gomez, L. (1997) Historia del currículo. Madrid.

Alles, M. (1997) Doscientos modelos de currículo. Argentina.

Burgos. (1981) Fundamentos generales del currículo. Editalaser, Bogotá.

Lafrancesco, G. (2003) Plan curricular. Escuela transformadora. Bogotá.

Guerrero, L. (1999) Plan curricular Segunda Edición Santa Fé.

Magendzo, K (2000) Currículo Educación para la democracia. PIIE Chile. 